\title{
Comparison Between Capsular and Transossous Suture Techniques for Treatment of Triangular Fibrous Cartilage Complex Injuries with Distal Radio-Ulnar Joint Instability
}

\section{Ryosuke Kakinoki ${ }^{1 *}$, Ryosuke Ikeguchi' ${ }^{2}$, Souichi Ohta ${ }^{2}$, Haruhiko Nishichi ${ }^{1}$, Hiroki Tanaka ${ }^{1}$, Kazuhiro Ohtani ${ }^{1}$ and Masao Akagi ${ }^{1}$}

${ }^{1}$ Department of Orthopedic Surgery, Kindai University Hospital, Japan

${ }^{2}$ Department of Orthopedic Surgery, Graduate School of Medicine, Kyoto University, Japan

*Corresponding Author: Ryosuke Kakinoki, Department of Orthopedic Surgery, Kindai University Hospital, Japan.
Received: November 30, 2020

Published: December 10, 2020

(C) All rights are reserved by Ryosuke

Kakinoki., et al.

\begin{abstract}
We use the capsular suture (CS) and trans-osseous suture (OS) techniques to treat patients with triangular fibrous cartilage complex (TFCC) injury associated with distal radio-ulnar joint (DRUJ) instability. The purpose of this study was to compare the outcomes between the two techniques with a minimum of 2 years of follow-up and to identify whether any pre-operative assessment factors correlated with post-operative Disability of the Arm, Shoulder and Hand (DASH) scores.

This study included 46 patients with TFCC injury with manually reducible DRUJ instability. Arthroscopic CS ( $\mathrm{n}=26)$ or OS (n = 20) was performed. Arcs of the wrist extension-flexion and radio-ulnar deviation, forearm supination-pronation, grip strength, DRUJ instability, and DASH and visual analogue scale scores for wrist pain were assessed pre-operatively and at the final follow-up. Spearman's correlation coefficients were calculated between the pre-operative assessments and the DASH scores at the final follow-up.

The CS and OS techniques provided good stability of the DRUJ, increase in grip strength, and decrease in wrist pain and DASH scores. The results did not differ significantly between the CS and OS groups. Only the pre-operative DASH score correlated with the DASH score at the final follow-up.

The results did not differ significantly between the CS and OS techniques performed on manually reducible TFCC injuries. Patients with a higher pre-operative DASH score were more likely to have a higher DASH score at the final follow-up.
\end{abstract}

Keywords: TFCC, DRUJ; Capsular Suture; Transosseous Suture; Ulna Fovea

\section{Abbreviations}

CS: Capsular Suture; OS: Trans-Osseous Suture; TFCC: Triangular Fibrous Cartilage Complex; DRUJ: Distal Radio-Ulnar Joint; DASH: Disability of the Arm, Shoulder and Hand; VAS: Visual Analogue Scale.

\section{Introduction}

Triangular fibrocartilage complex (TFCC) injury at the ulnar fovea is followed by distal radio-ulnar joint (DRUJ) instability, which often leads to pain and functional impairment in the affected wrist. The deep layer of the TFCC plays an important role in DRUJ stability. Because the TFCC attaches to the ulnar fovea, from the anatomical point of view, it is reasonable to re-attach the avulsed TFCC fragment to the ulnar fovea [1-6]. However, arthroscopic re-attachment of the TFCC to the ulnar fovea is complicated. Several guiding devices have been invented $[3,4]$ or an X-ray fluoroscopic examination can be used during surgery [6]. Creation of bone tunnels through the distal ulna epiphysis is not advantageous in young patients because of their open epiphyses. Researchers have reported that 
91-93\% of patients who undergo arthroscopic peripheral repair of the TFCC obtain satisfactory outcomes [7-10]. However, controversy remains in the results of cadaveric studies having compared the biomechanical strength of the TFCC repaired using the capsular suture (CS) method with that using trans-osseous bony re-attachment $[11,12]$.

The purposes of this study were to compare the outcomes between the arthroscopic-assisted CS [7,9] and trans-osseous suture (OS) [6] approaches for repairing TFCC injury with manually reducible DRUJ instability and to identify any pre-operative assessment factors that could predict Disability of the Arm, Shoulder and Hand (DASH) scores at least 2 years after TFCC repair.

\section{Materials and Methods \\ Cohort demography}

We performed a retrospective chart review of patients who had been diagnosed as having a TFCC injury at the ulnar fovea attachment in the hemi-lateral wrist using the wrist MRI or arthrograms and underwent arthroscopic TFCC repair operations in our hospital from 2014-2018. Additional inclusion criteria of this study were as follows. All patients were older than 14 years and followed up for $\geq 2$ years after surgery. All patients demonstrated the DRUJ instability in the affected wrists. All patients had no other pathological condition in the upper extremities except the hemi-lateral TFCC injury before surgery and during the follow-up period. In patients having had any problems such as operations, injuries or diseases in the upper limbs after the TFCC operations, the data recorded just before the occurrence of the problems were regarded as the final follow-up outcomes.(Within two years after the TFCC operations, one patient each of the OS and CS groups underwent ulnar shortening operations on the affected upper limb and one patient received the distal radius fracture in the unaffected wrist. These three patients were removed from the study.) In plain X-ray examinations, no osteoarthritic changes were seen in the DRUJ. The ulnar variance was set to be less than $2 \mathrm{~mm}$, based on previous studies showing that the outcomes of arthroscopic TFCC repair in patients with an ulnar variance greater than $+2 \mathrm{~mm}$ are unsatisfactory $[3,5]$. If a patient had ulnar head subluxation or dislocation, it was reducible manually. The operation modes, the CS or OS procedure were determined by patients' preference during the informed consent of the operations. Finally, 26 patients in the CS group and 20 in the OS group (21 men and 25 women) were included in this study. The demographic data of the patients are shown in Table 1 . The dominant hand was involved in 27 patients (16 in the CS group and 11 in the OS group). This study was approved by the ethical committee of our university hospital.

\section{Surgery}

With the patient under general anaesthesia, the affected wrist was distracted using a traction tower. A standard arthroscopic examination of the radio-carpal joint was performed through the 3-4 and 4-5 portals. For all patients, TFCC injury at the ulnar fovea was confirmed arthroscopically as a loss of normal tension of the TFCC (normal trampoline effect) and abnormal displacement of the TFCC with probing.

In the CS group, a $1.5 \mathrm{~cm}$-long palmar skin incision was made from the proximal margin of the pisiform along the flexor carpi ulnaris tendon, the palmar ulno-carpal joint (UCJ) was exposed by retracting the flexor carpi ulnaris tendon ulnarly, and the digital flexor tendons and the ulnar neurovascular bundle radially. Using a $7 \mathrm{~cm}$-long 21-gauge needle containing a 2-0 braid polyester or monofilament nylon suture in the barrel, we created an inside-out mattress suture through the palmar margin of the TFCC (a portion of the palmar radio-ulnar ligament) and the palmar UCJ capsule, referring to the technique described by Skie., et al. [10]. The suture was tied over the palmar joint capsule through the palmar skin incision (Figure 1a left). This procedure was repeated 1-3 times (Figure 1 right upper).

The dorsal CS procedure was performed using a $1.5 \mathrm{~cm}$-long longitudinal skin incision along the extensor carpi ulnaris (ECU) tendon over the ulna head. The ECU tendon (Figure $1 \mathrm{~b}$ left) was exposed and retracted ulnarly. Using a $3 \mathrm{~cm}$-long 21-gauge needle with a 3-0 nylon lasso suture (the first needle) inserted from the 6R portal and another $3 \mathrm{~cm}$-long 21-gauge needle containing a 2-0 monofilament nylon suture (the second suture) inserted parallel to the first needle through the ECU sub-sheath (Figure 1c left), we created an outside-in mattress suture (Figure $1 \mathrm{~d}$ left) through the ECU sub-sheath, the dorsal joint capsule and the dorsal portion of the TFCC, referring to the technique described by Whipple and Geissler [7] (Figure 1 right lower). 

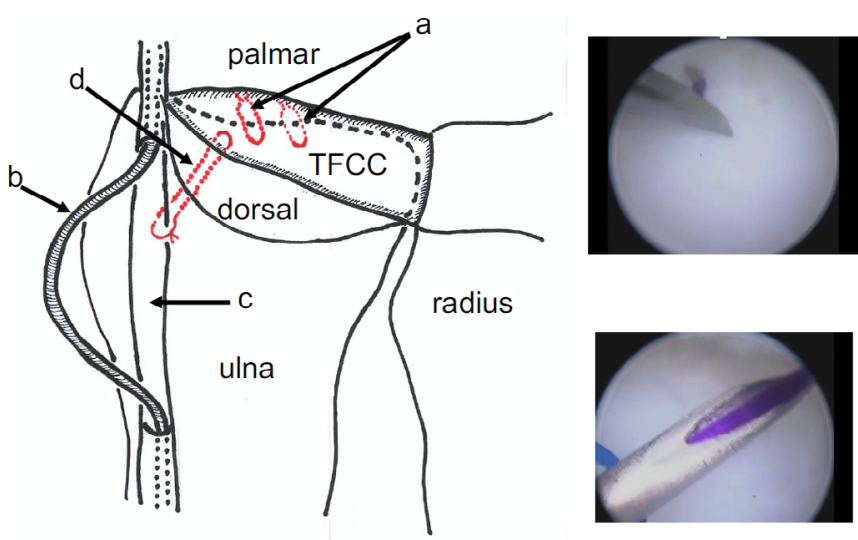

Figure 1: Left: a schematic diagram of the CS technique. Right upper: an intra-operative photo of the palmar peripheral capsular suture with an inside-out fashion. A $7 \mathrm{~cm}$-long $21 \mathrm{G}$ needle containing a 2-0 suture was being passed through the palmar periphery of the TFCC. Right lower; an intra-operative photo of the dorsal peripheral capsular suture with an outside-in fashion. A $3 \mathrm{~cm}$-long needle with a 2-0 monofilament nylon suture was passed through a 3-0 lasso suture. a Palmar peripheral sutures. b Extensor carpi ulnaris tendon. c Extensor carpi ulnaris sub-sheath. d Dorsal peripheral suture.

In the OS group, the operation was performed similarly to that described by Iwasaki., et al. [6]. A bone tunnel (Figure 2a left) was created using a screw with a $2.9 \mathrm{~mm}$ diameter from the lateral ulnar metaphysis to the ulnar fovea with X-ray fluoroscopic assistance. A 3 cm-long-21-gauge needle with a 3-0 nylon lasso suture and another $3 \mathrm{~cm}$-long-21-gauge needle with a 2-0 braid polyester suture were passed through the TFCC about $2 \mathrm{~mm}$ apart through the ulnar bone tunnel. A mattress suture with a 2-0 braid polyester suture was created using an outside-in fashion (Figure 2 right) to approximate the ulnar side of the TFCC to the ulna fovea through the bone tunnel (Figure $2 \mathrm{~b}$ left). If peripheral tears of the TFCC were found to be associated with the ulnar fovea injury, 3-0 absorbable monofilament sutures were added between the joint capsule and the TFCC margin across the TFCC tears using the inside-out or outside-in techniques described above.

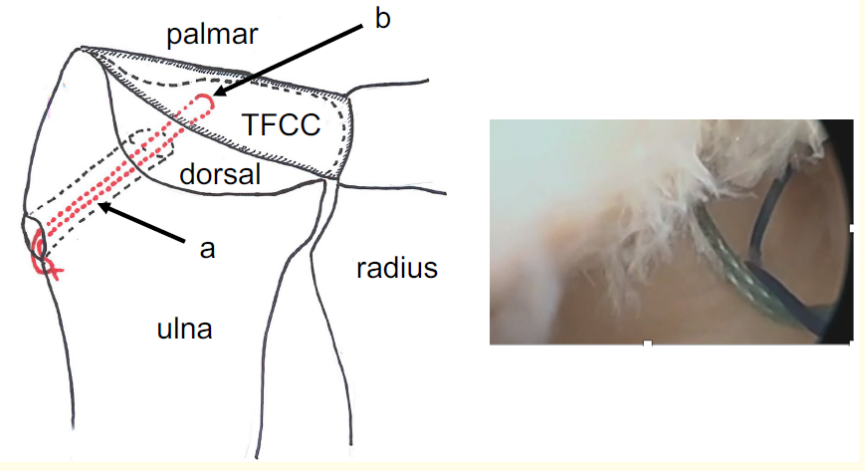

Figure 2: Left; a schematic diagram of the OS technique. Right: an intra-operative photo of the trans-osseous suture with an outside-in fashion. A 2-0 braid polyester suture was passed through a

3-0 lasso suture. a ulnar bone tunnel; b trans-osseous suture.

Each patient received long-arm cast fixation for 4 weeks after surgery and wore a soft wrist splint permitting active mild wrist motion for the next 2 weeks. The patients received a hand therapy programme starting 6 weeks after surgery and were allowed to restart sports activity 12 weeks after surgery.

\section{Assessments}

The arcs of the wrist in extension-flexion and radio-ulnar deviation, and forearm supination-pronation, grip strength, instability of the DRUJ, DASH score and visual analogue scale (VAS) score for wrist pain were assessed before surgery and at the final follow-up. Dorsal or palmar instability of the ulnar head was assessed in both wrists using the test described by Kleinman., et al. [2] and scored as 0 , no instability; 1 , instability similar to or less than that of the unaffected wrist; 2 , greater instability than the unaffected wrist with the end-point; and 3, greater instability than the unaffected wrist with no end-point. The DRUJ instability score was expressed as the sum of scores for the dorsal and palmar instabilities of the ulna. The grip strength and arc of the wrist and forearm were expressed as percentages relative to the unaffected limb. The ulnar variance was measured on a plain X-ray film (postero-anterior view) of the wrist in the neutral position taken at the initial visit to our clinic. The variance was classified as positive, neutral or negative variance. 
Comparison Between Capsular and Transossous Suture Techniques for Treatment of Triangular Fibrous Cartilage Complex Injuries with Distal Radio-Ulnar Joint Instability

\section{Statistics}

The male-to-female ratio, ratios for ulnar variance and the presence of traumatic episodes were compared between the CS and OS groups using the chi-squared test. Bivariate analysis was performed to compare the assessment results between the CS and OS groups, and between before surgery and at the final follow-up. To identify the prognostic pre-operative factors that predicted the post-operative DASH scores, Spearman's correlation coefficients were calculated for the DASH scores at the final follow-up and the pre-operative values for each assessment. A correlation coefficient $>0.4$ was considered to indicate a significant correlation. $\mathrm{p}<.05$ was accepted as significant.

\section{Results and Discussion}

Among the 46 patients, one patient in the OS group exhibited temporal ulnar nerve palsy post-operatively, but this had recovered completely within 3 months. One patient in the CS group and two in the OS group complained of complex regional pain syndromelike symptoms after surgery. One of these three patients returned to their original job within 6 months after surgery, the second patient's job was converted to light work, and the third patient was not yet working at the final follow-up. All patients except these latter two had returned to their original job 3-6 months after surgery.

In the CS group, all patients received palmar capsular sutures (mean suture number: 1.4; range: 1-2), and 21 patients received dorsal capsular sutures (mean suture number: 0.8; range: 0-1). 20 patients received one suture in the palmar periphery and two underwent the single dorsal peripheral sutures using 3-0 absorbable sutures. In the OS group, the number of the palmar periphery sutures is one in 10 patients and two in six patients and two patients received one suture in the dorsal periphery. Arthroscopic debridement of the disc portion of the TFCC was performed on four patients in the CS group and three patients in the OS group.

\section{Demographics of the patients in the two surgical groups}

The patients' demographic variables for the CS and OS groups are shown in table 1 . None of these variables differed significantly between the two groups.

\begin{tabular}{|l|c|c|c|}
\hline \multicolumn{1}{|c|}{ Groups } & CS Group & OS Group & p value \\
\hline Number & 26 & 20 & - \\
\hline Age (Years) & $\begin{array}{c}30 \pm 13.3 \\
(25.0-35.7)\end{array}$ & $\begin{array}{c}33 \pm 13.5 \\
(26.5-39.0)\end{array}$ & n.s \\
\hline Sex (Male/Female) & $15 / 11$ & $10 / 10$ & n.s \\
\hline Ulnar Variance (N/0/P) & $1 / 10 / 15$ & $1 / 8 / 11$ & n.s \\
\hline $\begin{array}{l}\text { Episode of the Wrist } \\
\text { Injury (U/K) }\end{array}$ & $9 / 17$ & $7 / 13$ & n.s \\
\hline $\begin{array}{l}\text { Onset-operation } \\
\text { Interval (Month) }\end{array}$ & $3.8 \pm 2.0$ & $4.7 \pm 2.6$ & n.s \\
\hline $\begin{array}{l}\text { Follow-up Period } \\
\text { (Month) }\end{array}$ & $\begin{array}{c}30.3 \pm 7.4 .4 \\
(27.3-33.4)\end{array}$ & $\begin{array}{c}30.6 \pm 7.0 \\
(27.3-33.8)\end{array}$ & n.s \\
\hline
\end{tabular}

Table 1: Comparison of Demographic Data between CS and OS groups.

Note. Ulnar variance: N: Negative Variance; 0: Neutral Variance; P: Positive Variance; Episode of the wrist Injury: U: Unknown Episode; K: Known Episode.

Pre-operative and final follow-up assessments of the two surgical groups

The values at the pre-operative and final follow-up assessments for both surgical groups are shown in tables 2 and 3, respectively. None of the assessment results differed significantly between the two groups before surgery or at the final follow-up.

\begin{tabular}{|c|c|c|c|}
\hline Groups & CS Group & OS Group & p value \\
\hline $\begin{array}{c}\text { Extension-flexion } \\
\text { Arc }\end{array}$ & $\begin{array}{c}91.9 \pm 16.0 \\
(85.5-98.4)\end{array}$ & $\begin{array}{c}93.0 \pm 9.1 \\
(88.7-97.3)\end{array}$ & n.s \\
\hline Radial-ulnar & $86.8 \pm 17.4$ & $86.1 \pm 16.2$ & n.s \\
Deviation Arc & $(79.8-93.8)$ & $(78.5-93.6)$ & \\
\hline Pronation- & $95.5 \pm 8.1$ & $92.8 \pm 14.1$ & n.s \\
supination Arc & $(92.2-98.8)$ & $(86.1-99.4)$ & \\
\hline Grip Strength & $76.0 \pm 18.6$ & $80.7 \pm 18.4$ & n.s \\
\hline DRUJ Instability & 4 & 4 & n.s \\
Score & & & n.s \\
\hline VAS Score & $52.7 \pm 13.3$ & $56.3 \pm 17.4$ & n.s \\
& $(47.3-58.1)$ & $(48.1-64.4)$ & \\
\hline DASH Score & $33.6 \pm 16.5$ & $36.8 \pm 23.1$ & $(26.0-47.6)$ \\
& $(27.0-40.3)$ & & \\
\hline
\end{tabular}

Table 2: Statistical Comparison of the Preoperative Examinations Between CS and OS Groups.

Note: Mean \pm standard deviation, except the DRUJ instability. Median values were expressed in the DRUJ instability scores. Values in parentheses meant the 95\% Confidential Intervals. Wrists and forearms arcs and grip strength of the affected upper limbs were expressed as percentages of those of the contralateral healthy upper limbs. 
Comparison Between Capsular and Transossous Suture Techniques for Treatment of Triangular Fibrous Cartilage Complex Injuries with Distal Radio-Ulnar Joint Instability

35

\begin{tabular}{|c|c|c|c|}
\hline Groups & CS Group & OS Group & p value \\
\hline Extension-flexion Arc & $96.2 \pm 7.8$ & $96.0 \pm 6.5$ & n.s \\
& $(93.1-99.4)$ & $(93.0-99.0)$ & \\
\hline Radial-ulnar Deviation & $91.1 \pm 15.2$ & $93.3 \pm 14.3$ & n.s \\
Arc & $(85.0-97.2)$ & $(86.6-99.9)$ & \\
\hline Pronation-supination & $98.2 \pm 3.4$ & $96.7 \pm 4.9$ & n.s \\
Arc & $(96.9-99.6)$ & $(94.4-98.9)$ & \\
\hline Grip Strength & $89.6 \pm 15.2$ & $90.3 \pm 12.8$ & n.s \\
& $(83.5-95.7)$ & $(84.3-96.3)$ & \\
\hline DRUJ Instability Score & 1 & 1 & n.s \\
\hline VAS Score & $11.6 \pm 12.7$ & $12.1 \pm 10.7$ & n.s \\
& $(6.5-16.7)$ & $(7.1-17.1)$ & \\
\hline DASH Score & $7.5 \pm 9.0$ & $5.6 \pm 6.8$ & n.s \\
& $(3.9-11.2)$ & $(2.5-8.8)$ & \\
\hline
\end{tabular}

Table 3: Statistical Comparison of the Postoperative Examinations Between CS and OS Groups.

Note: Mean \pm standard deviation, except the DRUJ instability. Median values were expressed in the DRUJ instability scores. Values in parentheses meant the $95 \%$ Confidential Intervals. Wrists and forearms arcs and grip strength of the affected upper limbs were expressed as percentages of those of the contralateral healthy upper limbs.

Comparison between assessment values before surgery and at the final follow-up of all patients including the CS and OS groups

The mean or median values for each assessment item before surgery and at the final follow-up for all patients, including the CS and OS groups, are shown in table 4 . The mean arcs of the wrist and forearm did not change after surgery. The mean grip strength increased significantly. The median DRUJ instability score and the mean VAS and DASH scores decreased significantly after surgery. Twenty-three of the 46 patients $(13 / 26$ in the CS group and 10/20 in the OS group) still complained of some disability in the affected upper extremities (DASH scores ${ }^{3} 5$ ) at the final follow-up (data were not shown).

Pre-operative assessments for predicting the final DASH score

Among the pre-operative assessments, only the DASH score before surgery was moderately correlated $(r=0.442, p=0.0021)$

\begin{tabular}{|c|c|c|c|}
\hline Time of Examination & $\begin{array}{c}\text { Before } \\
\text { Surgery }\end{array}$ & $\begin{array}{c}\text { After } \\
\text { Surgery }\end{array}$ & p value \\
\hline Extension-flexion Arc & $92.4 \pm 13.3$ & $96.1 \pm 7.2$ & n.s \\
& $(88.4-96.3)$ & $(94.0-98.3)$ & \\
\hline Radial-ulnar Deviation & $86.5 \pm 16.7$ & $92.0 \pm 14.7$ & n.s \\
Arc & $(81.5-91.4)$ & $(87.7-96.4)$ & \\
\hline Pronation-supination & $94.3 \pm 11.1$ & $97.5 \pm 4.1$ & n.s \\
Arc & $(91.0-97.6)$ & $(96.3-98.8)$ & \\
\hline Grip Strength & $77.1 \pm 17.5$ & $89.0 \pm 12.4$ & 0.0001 \\
& $(71.9-82.3)$ & $(85.3-92.6)$ & \\
\hline DRUJ Instability Scores & 4 & 1 & $<0.0001$ \\
\hline VAS Score & $54.2 \pm 15.1$ & $11.8 \pm 11.7$ & $<0.0001$ \\
& $(49.7-58.7)$ & $(8.3-15.3)$ & \\
\hline DASH Score & $35.0 \pm 19.5$ & $6.7 \pm 8.1(4.3-$ & $<0.0001$ \\
& $(29.2-40.8)$ & $9.1)$ & \\
\hline
\end{tabular}

Table 4: Statistical Comparisons Between the Preoperative and Postoperative Examinations in All Patients (Including CS and OS groups).

Note: Mean \pm standard deviation, except the DRUJ instability scores. Median values were expressed in the DRUJ instability scores. Values in parentheses meant the $95 \%$ Confidential Intervals. Wrists and forearms arcs and grip strength of the affected upper limbs were expressed as percentages of those of the contralateral healthy upper limbs.

with that at the final follow-up (Table 5). Receiver-operating characteristic curve analysis showed that patients with a pre-operative DASH score ${ }^{3} 35.3$ were likely to have a DASH score ${ }^{3} 5$ at the final follow-up (sensitivity $=65.2 \%$, specificity $=78.3 \%$, positive predictive value $=75.0 \%$, negative predictive value $=69.2 \%$, area under the curve $=0.759$ with $95 \%$ confidence interval $=0.610$ to 0.873 ).

The CS and OS procedures performed on TFCC injury with manually reducible DRUJ instability significantly increased stability of the DRUJ and grip strength and decreased wrist pain, as measured by the VAS and DASH scores, more than 2 years after surgery. The results did not differ significantly between the two procedures. About half of the patients in both groups still complained of some disability in the affected upper extremities (DASH score ${ }^{3} 5$ ) at the final follow-up. The DASH score before surgery correlated moderately with that at the final follow-up. About $75 \%$ of patients with a DASH score $>35$ before surgery were likely to have a score ${ }^{3} 5$ at 2 years after surgery. 
Comparison Between Capsular and Transossous Suture Techniques for Treatment of Triangular Fibrous Cartilage Complex Injuries with Distal Radio-Ulnar Joint Instability

\begin{tabular}{|c|c|c|}
\hline Preoperative Assessment & r value & p value \\
\hline Age & 0.312 & 0.0348 \\
\hline Sex & -0.167 & n.s. \\
\hline Affected side & -0.085 & n.s. \\
\hline Ulnar variance & 0.1206 & n.s. \\
\hline $\begin{array}{c}\text { Known Onset of the Wrist } \\
\text { Pain }\end{array}$ & -0.211 & n.s. \\
\hline Onset-operation Interval & -0.126 & n.s. \\
\hline F/u period & -0.05 & n.s. \\
\hline Operation Mode & -0.101 & n.s. \\
\hline Extension-flexion Arc & -0.202 & n.s. \\
\hline Radial-ulnar Arc & -0.363 & 0.0133 \\
\hline Pronation-supination Arc & -0.016 & n.s. \\
\hline Grip Strength & -0.383 & 0.0086 \\
\hline DRUJ Instability & 0.142 & n.s. \\
\hline VAS Score & 0.099 & n.s. \\
\hline DASH Score & 0.442 & 0.0021 \\
\hline \multicolumn{2}{|l}{} \\
\hline
\end{tabular}

Table 5: Spearman's coefficients between DASH score at the final follow-up and the preoperative assessments.

The CS procedure is not an anatomical repair of the TFCC. Several authors have placed a premium on the re-attachment of the TFCC to the ulnar fovea in the treatment of TFCC injuries with DRUJ instability [1-6]. However, others have reported satisfactory outcomes of arthroscopic CS of TFCC injuries with DRUJ instability [7-10]. For the biomechanical comparison, the choice between capsular and trans-osseous sutures remains controversial. Johnson., et al. reported that the trans-osseous TFCC repair provides significantly less displacement and greater stiffness of the DRUJ than the capsular repair [11]. By contrast, Ruch., et al. found no significant difference in the biomechanical stability of the DRUJ between the capsular and trans-osseous suture techniques [12], although the techniques used to repair the TFCC in these studies differed.

Because the ulnar subluxation or dislocation was manually reduced in our study, some structures that stabilise the TFCC besides the deep ligamentous portion of the TFCC (e.g. the superficial ligamentous portions of the TFCC [2], distal oblique band of the interosseous ligament $[13,14]$, or ECU sub-sheath [14]), may have remained functional. The CS procedure may have increased the tension within these structures, which may have increased the TFCC tension and provided DRUJ stability. Our findings suggest that re-attachment of the TFCC to the ulnar fovea may not always be necessary to repair TFCC injuries with manually reducible DRUJ instability. The CS may be strongly indicated for young patients whose ulnar epiphyses are still open.

In this study, the patients' ulnar variance was set to be less than $+2 \mathrm{~mm}$. We performed the ulnar shortening on patients having ulnar fovea TFCC injury with the ulnar variance $\geq+2 \mathrm{~mm}$., because previous authors mentioned that the outcomes of arthroscopic TFCC repairs in patients with the ulnar variance greater than $+2 \mathrm{~mm}$ were unsatisfactory $[3,5]$. In fact, the ulnar variance was not correlated with the final follow-up outcomes in the current cohort having ulnar variance $<2 \mathrm{~mm}$ (Table 4 ). As the previous authors mentioned, TFCC suture should be indicated for patients having ulnar variance $<2 \mathrm{~mm}$. However, two patients having positive ulnar variance (one each in the CS and OS groups) were removed from the current study because of having undergone the ulnar shortening within two years after TFCC repairs due to no subsidence or recurrence of the writ pain after the TFCC repairs. Patients with negative or neutral ulnar variance may be more indicated for TFCC repairs than those with positive ulnar variance despite $<+2 \mathrm{~mm}$.

This study has some limitations. First, this is the retrospective study using the chart review. Second, the results of this study are applicable to patients with TFCC injury associated with manually reducible DRUJ subluxation or dislocation. Third, the study does not provide a strict comparison of outcomes between the CS and OS techniques for TFCC because some capsular sutures were added to the trans-osseous suture in some patients in the OS group.

\section{Conclusions}

When TFCC injuries associated with manually reducible DRUJ subluxation or dislocation, the outcomes of the CS and OS procedures did not differ significantly at the final follow-up more than two years after surgery and both procedures significantly increased stability of the DRUJs and grip strength and decreased the wrist pain and disabilities. Approximately $75 \%$ of the patients having the DASH scores $>35$ before surgery may likely have the scores $\geq 5$ two years after surgery.

\section{Conflict of Interest}

We received no funds or financial support from anywhere with regard to this study. On behalf of all authors, the corresponding 
author states that there is no conflict of interest. This study was approved by the ethical committee in Kindai University Hospital (R02-034). This study was informed of all patients in our hospital using an opt-out announcement. We declared to all patients that all data were anonymized and analysed to protect the privacy.

\section{Bibliography}

1. Hermansdorfer JD and Kleinman WB. "Management of chronic peripheral tears of the triangular fibrocartilage complex". Journal of Hand Surgery Am 16.2 (1991): 340-346.

2. Kleinman WB. "Stability of the distal radioulnar joint: biomechanics, pathophysiology, physical diagnosis and restoration of function what we have learned in 25 years". Journal of Hand Surgery Am 32.7 (2007): 1086-1106.

3. Nakamura T., et al. "Repair of foveal detachment of the triangular fibrocartilage complex: open and arthroscopic transosseous techniques". Hand Clinic 27 (2011): 281-290.

4. Fujio K. "Arthroscopic management of triangular fibrocartilage complex foveal injury”. Hand Clinic 33.4 (2017): 619-624.

5. Abe Y., et al. "Midterm results after open versus arthroscopic transosseous repair for foveal tears of the triangular fibrocartilage complex". Journal of Wrist Surgery 7.4 (2018): 292-297.

6. Iwasaki N and Minami A. "Arthroscopically assisted reattachment of avulsed triangular fibrocartilage complex to the fovea of the ulnar head". Journal of Hand Surgery Am 34.7 (2009): 1323-6.

7. Whipple TL and Geissler WB. "Arthroscopic management of wrist triangular fibrocartilage complex injuries in the athlete". Orthopedics 16.9 (1993): 1061-7

8. Trumble TE., et al. "Isolated tears of the triangular fibrocartilage: management by early arthroscopic repair". Journal of Hand Surgery Am 22.1 (1997): 57-65.

9. Corso SJ., et al. "Arthroscopic repair of peripheral avulsion of the triangular fibrocartilage complex of the wrist: a multicenter study". Arthroscopy 13.1 (1997): 78-84.
10. Skie MC., et al. "Operative technique for inside-out repair of the triangular fibrocartilage complex". Journal of Hand Surgery Am 22.5 (1997): 814-817.

11. Johnson JC., et al. "Biomechanical analysis of capsular repair versus Arthrex TFCC ulnar tunnel repair for triangular fibrocartilage complex tears". Hand (NY) 14.4 (2019): 547-553.

12. Ruch DS., et al. "Biomechanical comparison of transosseous and capsular repair of peripheral triangular fibrocartilage tears". Arthroscopy 19.4 (2003): 391-396.

13. Moritomo H. "The distal interosseous membrane: current concepts in wrist anatomy and biomechanics". Journal of Hand Surgery Am 37.7 (2012): 1501-1507.

14. Stuart PR., et al. "The dorsopalmar stability of the distal radioulnar joint”. Journal of Hand Surgery Am 25.4 (2000): 689-699.

\section{Assets from publication with us}

- Prompt Acknowledgement after receiving the article

- Thorough Double blinded peer review

- Rapid Publication

- Issue of Publication Certificate

- High visibility of your Published work

Website: https://www.actascientific.com/

Submit Article: https://www.actascientific.com/submission.php Email us: editor@actascientific.com

Contact us: +919182824667 